\title{
An Efficient Routing Protocol on Adhoc Wireless Mobile Networks
}

\author{
R. Lal Raja Lokesh Babu, D. Pitchaiah, Prof. P. Prasanna Murali Krishna, \\ M.Ramana Reddy \\ M.tech II year, Dr.sgit, markapur, Associ.prof, Dr.SGIT,markapur, h.o.d of decs Dr.SGIT.markapur, \\ M.tech II year, Dr.SGIT.markapur
}

\begin{abstract}
Efficient routing protocols can provide significant benefits to mobile ad hoc networks in terms of both performance and reliability. Mobile Ad-hoc Network (MANET) is an infrastructure less and decentralized network which need a robust dynamic routing protocol In ad hoc network networks, in order to make them reliable and secure one need to analyze the routing mechanisms which are called as the MANET routing protocols and also transport protocols also required. In the research work, we have to do the investigation of mobile ad hoc network protocols like ad hoc on demand routing protocol AODV, DSDV and DSR protocol using the different TCP types like TCP Reno, TCP New Reno, TCP Vegas and our Analysis of the variants of TCP is based on these performance metrics: TCP Throughput, Average End-to-End delay, Packet Delivery Fraction and Energy Cost. This analysis will be useful in determining the better variant among TCP Protocols to ensure better data transfer, speed, and reliability and congestion control.
\end{abstract}

Keywords: MANET, AODV, DSR, DSDV, TCP Reno, New Reno, Vegas.

\section{INTRODUCTION}

The routing protocols in the MANET are our traditional networks, however routing protocols deals with the various challenges which is only because of the nodes mobility which more prone to the errors as compared to the wired networks.

Due to the dynamic mobility and routing between the mobile nodes, routes between the mobile nodes sometimes disappear and again back which resulted into the MANET routing mechanism more complicated as compared to the wired network. To finding the optimal communication route from source to destination is only basic and main goal of routing in MANET. Optimal path considers the other network factors as well such as latency, jitter, network overhead, throughput, communication cost and power in order to communicate between the source and destination without failure.

Due to mobility the communication paths are changing very frequently and hence network packets are not at all affected or even not changing the packet optimality and its uniformity.

There are mainly three categories of the mobile routing protocols such as proactive, reactive and hybrid routing protocols as shown following figure 1. There are many protocols which we are considering for the investigation and evaluation in the mobile ad hoc networks. But each of these routing protocols is focused on the certain aspects of simulation results TCP is not well suited for wireless networks especially in MANET; the performance of TCP degrades significantly due to the heavy packet and connection losses.

To overcome the problems of reliability, versions of TCP called TCP variants were developed especially for wireless ad hoc networks to provide reliable communication.

There are different network layer protocols for route discovery and maintenance in MANET but, the issue is the selection of suitable coupling of TCP variant over MANET routing protocol to provide reliable communication.

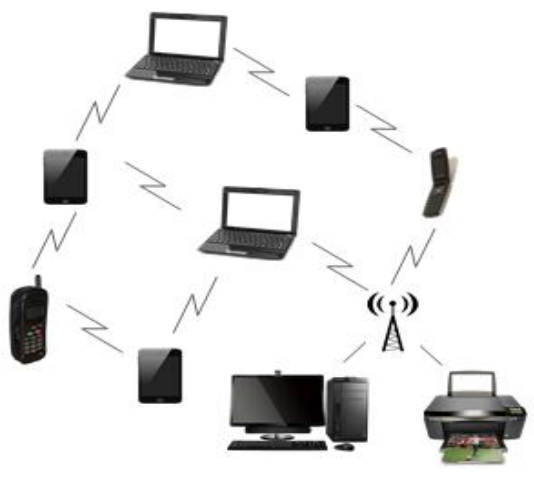




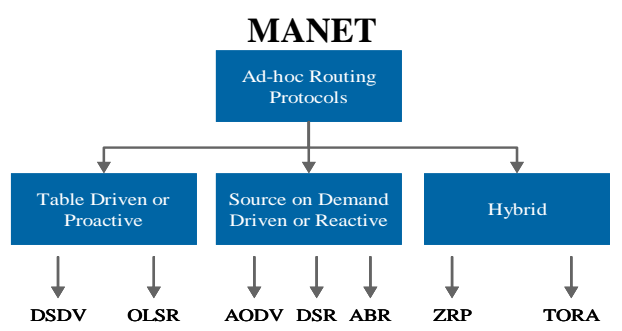

\section{STUdY OF DSDV AND AODV DSR ROUTING PROTOCOLS}

\section{A. Destination Sequenced Distance Vector (DSDV)}

DSDV is one of the most well known table-driven routing algorithms for MANETs. The DSDV routing algorithm is based on the number of hops to reach to the destination, sequence number of the classical Bellman-Ford Routing Algorithm with certain improvement [3]. Each and every mobile node maintains a routing table with all available destinations along with some more information [4].

Advantages of DSDV:

- DSDV was one of the early algorithms available. It is quite suitable for creating ad hoc networks with small number of nodes.

Disadvantages of DSDV

- DSDV requires a regular update of its routing tables, which uses up battery power and a small amount of bandwidth even when the network is idle

- Whenever the topology of the network changes, a new sequence number is necessary before the network reconverges; thus, DSDV is not suitable for highly dynamic networks

\section{B. Ad-hoc On-demand Distance Vector (AODV)}

Reactive protocols discover routes when it's required. If a node wishes to communicate with another node, it checks with its previous information for a valid route to the destination. If one route is found, the node uses that route for communication with the destination node. If route is not found, the source node starts a route discovery process by RREQ, to which either the destination node or one of the intermediate nodes sends a reply back to the source node with a valid route [5]. Less amount of information (mostly fixed packet size) is stored into routing packet unlike DSR routing protocol.

\section{Advantages of AODV:}

In AODV, route discovery process is in on demand, which is more efficient in dynamic nature of mobile ad-hoc network.

\section{Disadvantages:}

Due to on demand manner, it won't check route in periodic interval so transmission of data after discover the rote is taking some more delay, but due to dynamic nature of network this delay is not considerable.

\section{TCP}

\section{A. RENO}

The Reno TCP implementation retained the enhancements to Tahoe, but changed the Fast Retransmit operation to include Fast Recovery [Jac90]. This algorithm prevents the communication path from going empty after Fast Retransmit, because of that avoiding the need to Slow-Start to re-fill it after a single packet loss.

In Reno, the sender' s usable window becomes $\min ($ awin, $c w n d+n d u p)$ where awin is the receiver's advertised window, cwnd is the sender' s congestion window, and ndup is maintained at 0 until the number of dup ACKs reaches tcprexmtthresh, thenceforth tracks the number of duplicate ACKs. Thus, during Fast Recovery the sender "inflates" its window by the number of dup ACKs it has received, accordant with the observation that each dup ACK indicates some packet has been removed from the network and is now cached at the receiver. After entering

\section{B. TCP Vegas}

TCP Vegas was proposed by Brakmo et al. It has a very different congestion control algorithm compared to New Tahoe. TCP Vegas[10] in general controls its segment flow rate based on its estimate of the available network bandwidth. Among the many new features implemented in TCP Vegas, the most important difference between it and TCP Tahoe lies in its bandwidth estimation scheme. Studies on TCP Vegas have shown that Vegas achieve higher efficiency than Tahoe, causes fewer packet retransmissions 


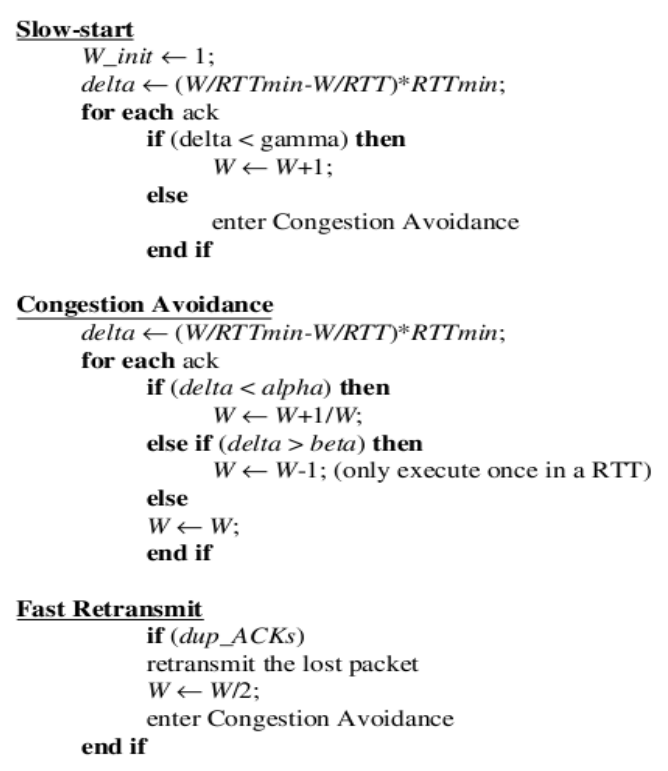

\section{New Reno:}

TCP New Reno defined by RFC 3782, advances retransmission during the fast recovery phase of TCP Reno. while fast recovery for every duplicate ACK that is returned to TCP New Reno, a new not sent packet from the end of the congestion window is sent, to keep the transmit window as full.

For each and every ACK that provides partial progress in the sequence space, sender assumes that the ACK points to a new hole and the next packet beyond the ACK ed sequence number is sent.

\section{TCP OVER MANET}

As a result of the improvement of wireless technology and the proliferation of handheld wireless terminals, now a day's have witnessed an ever-increasing popularity of wireless communication, ranging from wireless WLAN and WWANs to MANETs. In WLANs (e.g., the Wi-Fi technology) in WWANs (e.g. $2.5 \mathrm{G} / 3 \mathrm{G} / 4 \mathrm{G}$ cellular networks), mobile station communicate with an access point or a base station that connected to the wired networks. Patently, only one hop wireless link is needed for communications between a mobile host and a stationary host in wired networks. In counterpoint, there no fixed infrastructure such as base stations or access points a MANET. All nodes in a MANET are capable of moving independently and functioning as a router that discovers and maintains routes and forwards packets to other nodes. Thus, mobile ad-hoc networks are multi-hop wireless networks by nature.

MANETs are multi-hop wireless networks by nature. Note that MANETs may be connected at the edges to the wired Internet. Transmission control protocol (TCP) is a transport layer protocol which provides reliable end to end data delivery between end hosts in traditional wired network environment. In TCP, reliability is achieved by retransmitting lost packets. Each TCP sender maintains a running average of the estimated round trip delay and the average deviation derived from it. Lost packets will be retransmitted if the sender receives no acknowledgment within a certain timeout interval (e.g., the sum of smoothed round trip delay and four times the average deviation) or receives duplicate acknowledgments.

Unfortunately, wireless networks and wired networks are significantly different in terms of propagation delay, bandwidth and link reliability. The conditional relation of the difference is that packet losses are no longer mainly due to network congestion; that may well be due to some wireless specific reasons. As a matter of fact, in cellular networks or WLANs, most packet losses are due to high bit error rate in wireless channels and handoffs between two Base stations, while in MANETS, most packet losses are due to medium contention and route breakages, and also radio channel errors. Therefore, TCP performs well in wired networks; but it will suffer from serious performance degradation in wireless a network if it misinterprets such no congestion related losses as a sign of congestion and consequently invokes congestion control and avoidance process, as confirmed through analysis and extensive simulations carried out.

As TCP performance disintegrates more seriously in ad hoc networks compared to cellular networks or WLANs, we divide wireless networks into two large groups: first one is called one-hop wireless networks that include WLANs and cellular networks and the other is called multi-hop wireless networks that include MANETs.

To understand TCP behavior and improve TCP performance over MANET, given these wireless specific issues, considerable research has been carried out and many schemes have been suggested. As the research in this area is still active and many difficulties are still wide open. 
This chapter helps to pinpoint the primary causes for TCP performance degradation over wireless networks and cover the state of the art in the solutions, in hopes that readers can better understand the problems and hence propose better solutions based on the current ones.

\section{Packet delivery function:}

\section{PARAMETERS} destination.

Packet delivery function is the defined as number of packets successfully transmitted b/w source and

\section{End to End delay:}

Time duration b/w packet received and sending time is called as End to End delay

\section{Overhead:}

Number of extra packets such as routing packets called as overhead

\section{Convergence time:}

Time duration b/w route failure and route recovery is called as convergence time

\section{PERFormance EVAluation}

We studied the different network environment by using NS2 tool. In our project we simulated various network environments given as bellow table.

\begin{tabular}{|l|l|}
\hline Parameter name & Parameter value \\
\hline Area & 500 X 500, 700 X 700, 1600 X 1600 \\
\hline Coverage area & $86 \mathrm{~m}, 230 \mathrm{~m}, 410 \mathrm{~m}$ \\
\hline Routing protocol & AODV, DSR, DSDV \\
\hline Transport protocol & TCP--- Reno, New Reno, Vegas \\
\hline No. Of nodes & $8,10,50,100,200$ \\
\hline Speed & $5 \mathrm{~m} / \mathrm{s}, 10 \mathrm{~m} / \mathrm{s}, 20 \mathrm{~m} / \mathrm{s}$ \\
\hline MAC & MAC/802.11 \\
\hline
\end{tabular}

In our project we are comparing the different routing protocols along with TCP protocol in different network environment. Simulation result for packet delivery ratio is shown in the following graphs, TCP New Reno is mostly providing high performance with DSR and AODV but not in DSDV

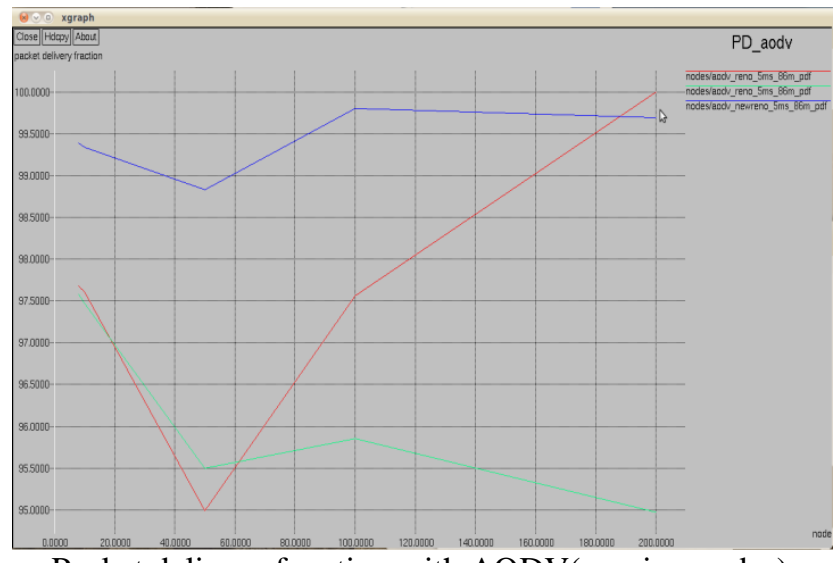

Packet delivery function with AODV(varying nodes)

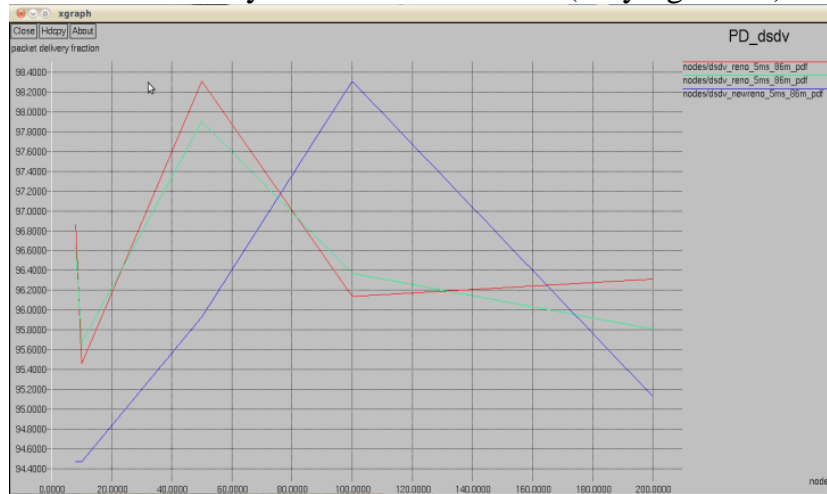

Packet delivery with DSDV (varying nodes)

In next comparison, we used variable as coverage area. In this result also TCP New reno is better than remaining TCP protocols 


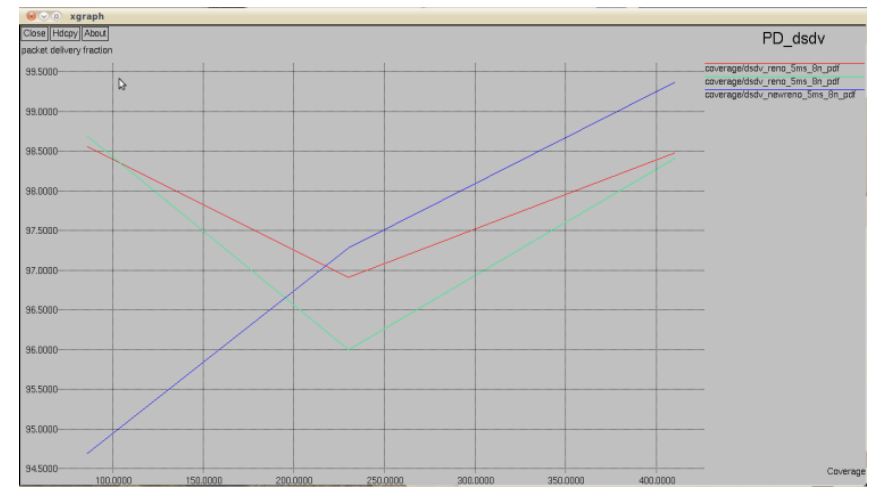

Packet delivery function with AODV(coverage)

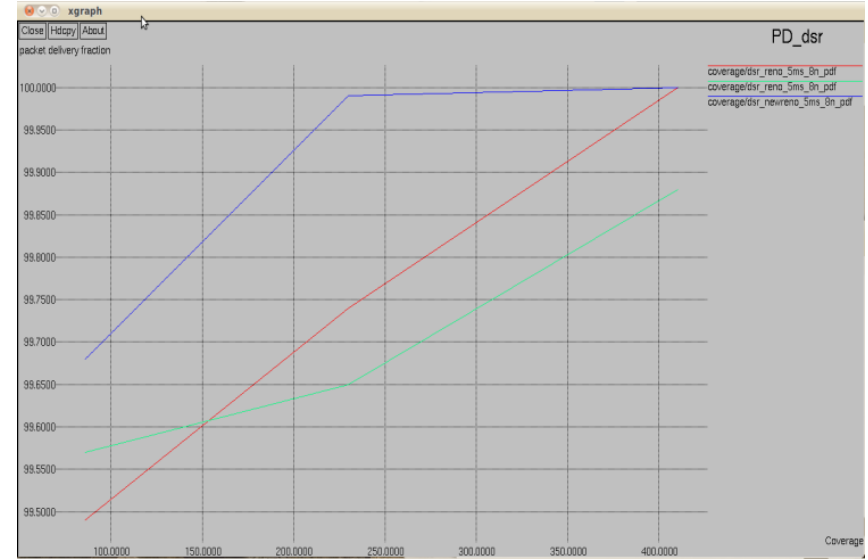

Packet delivery with DSR(coverage)

In End to End delay analysis, Reno providing constant delay in different network environment in all the routing protocol. But new Reno is proving less delay in 100 node environment for all routing protocols

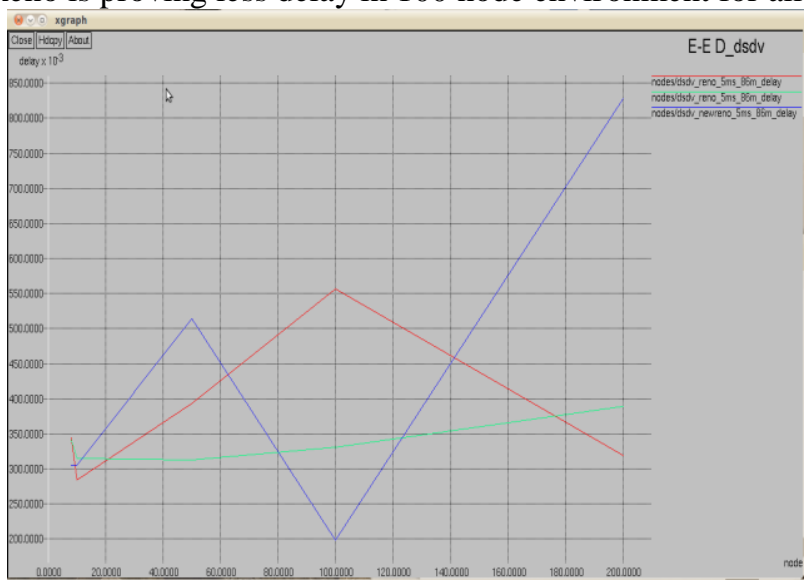

End to End delay with DSDV(nodes)

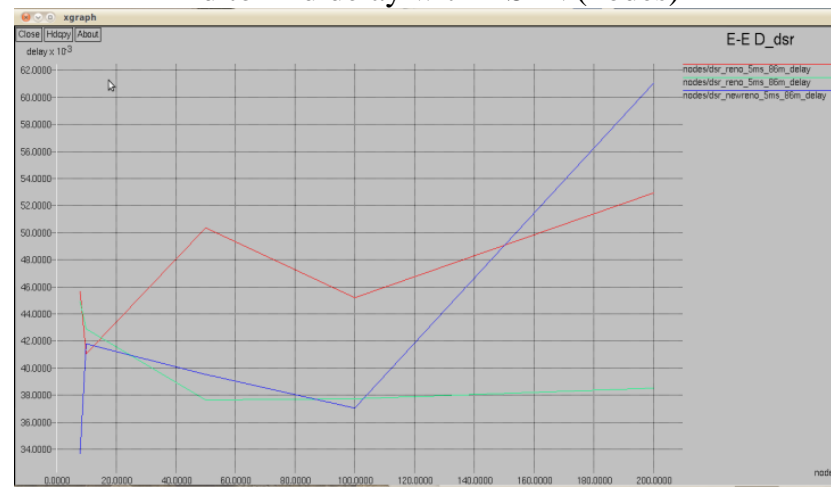




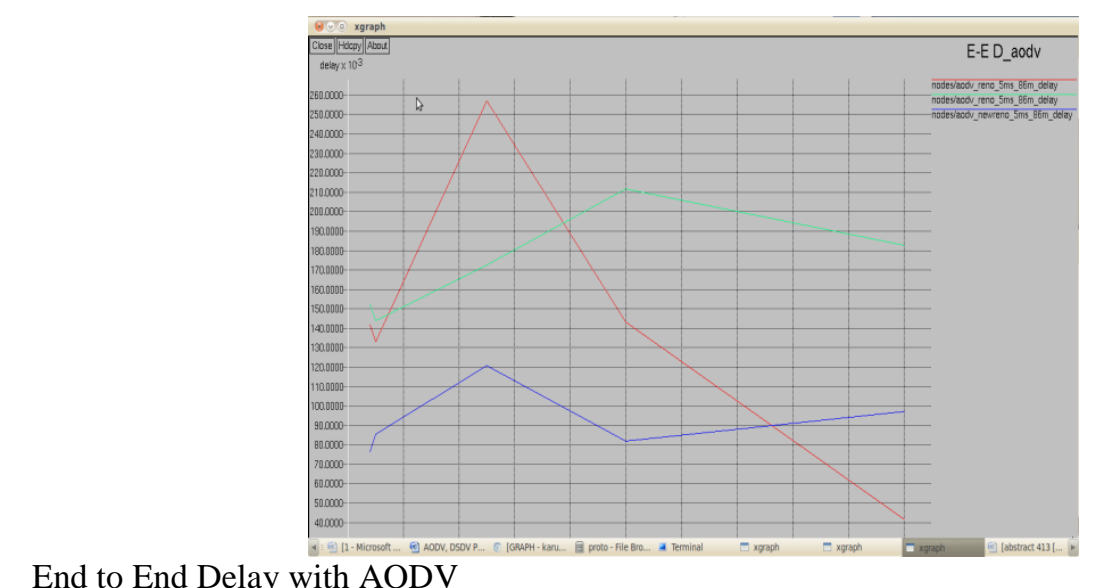

End to End Delay with AODV

In convergence time analysis, DSR providing less convergence time in most of the cases.

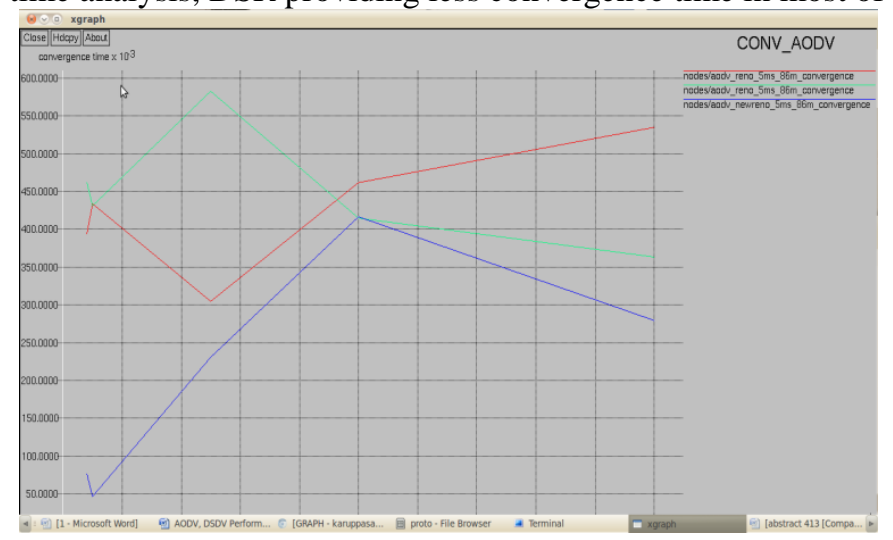

Convergence time with AODV(varying nodes)

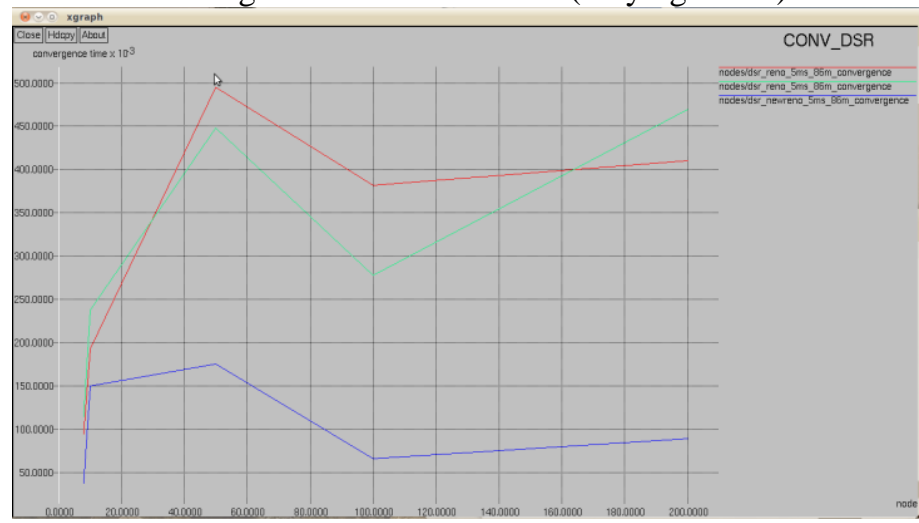

Convergence time with DSR(varying nodes)

VII. CONCLUSION:

This research work was based on the protocol investigation from the three main categories of MANET routing protocols such proactive routing protocol, reactive routing protocols with different TCP variants such as Vegas, Reno and New Reno. The protocols which we study and analyze are AODV, DSR, and DSDV from reactive routing protocols and proactive routing protocols respectively with varying the number nodes, data connections, network size. According to the results which are obtained in the results and discussion section by considering the throughput, delay, Our result shows that, the reaction of TCP in most cases could not be the right one: for example dealing with data packet losses due to link loss as if it was a strong congestion is proved to be an erroneous reaction. From that, we suggest that TCP should have a data packet loss classification algorithm in order to classify the reason of data packet losses and accordingly triggering the most appropriate data loss recovery algorithm strategy. The loss differentiation algorithms should have the ability to recognize the different data packet loss causes within wireless mobile ad-hoc networks (network congestion, wireless channel errors, and link loss) with a minimum computational overhead (i.e. without storing and maintaining too much state information). 


\section{Acknowledgment}

We wish to acknowledge the efforts of Pantech Solution Pvt Itd., India, for guidance which helped us work hard towards producing this research work.

\section{Reference}

[1]. David B. Johnson, David A. Maltz "Dynamic Source Routing in Ad Hoc Wireless Networks", Computer Science Department, Carnegie Mellon University, 1996.

[2]. Charles E. Perkins, “Ad-hoc On-Demand Distance Vector Routing”, 2000.

[3]. HOU-TSAN LEE*, FENG-Li LiAn, AND TING-CHUN FONG, “CONGESTION CONTROL OF TRANSMISSION CONTROL PROTOCOL BASED ON BANDWIDTH ESTIMATION”, JOURNAL OF THE CHINESE INSTITUTE OF ENGINEERS, VOL. 33, NO. 3, PP. 489-494 (2010)

[4]. J. Broch, D Johnson and D. Maltz , "The Dynamic Source Routing Protocol for Mobile Ad Hoc Networks (DSR)," IETF INTERNET-

[5]. DRAFT, 19 July 2004 C.E. PERKInS AND E.M. RoYer , “AD-HOC ON-DEMAND DiSTANCE VECTOR (AODV) ROUTING,” PROC. OF 2ND IEEE WORKSHOP ON MOBILE COMPUTING SYSTEMS AND APPLICATIONS, 1999

[6]. C.E. PERKINS, E.M. ROYER AND S.R. DAS , “AD HOC ON-DEMAND DISTANCE VECTOR (AODV) ROUTING,”, RFC 3561, JULY 2003

[7]. C.E. PERKINS AND P. BHAGWAT, "HIGHLY DYNAMIC DESTINATION SEQUENCED DISTANCE VECTOR ROUTING (DSDV) FOR MOBILE COMPUTERS,” PROC. OF ACM SIGCOMM'94, 1994

[8]. A. Boukerche, "Performance Evaluation of Routing Protocols For Ad Hoc Wireless Networks," MobiLe Networks AND APPLICATIONS, 2004

[9]. P. Johansson, T. Larsson, N Hedman, B Mielczarek And M.Degemark, "Scenario-Based Performance Analysis of Routing Protocols For Mobile AD-HOC Networks,” MOBICOM '99, 1999.

[10]. ANNAPURNA P PATIL, “CONVERGENCE TIME EVALUATION OF ALGORITHMS IN MANETs”, IJCSIS 2009 